\title{
Application of a modified optical fiber in targeted percutaneous laser disc decompression of lumbar disc herniation: A retrospective study
}

\author{
CHAO MENG ${ }^{1}$, YUJUN LI ${ }^{2}$, SHIJIE WANG ${ }^{1}$, JUNMIN YU $^{1}$, DEWEI KOU ${ }^{1}$ and CHUANSHENG LIU ${ }^{1}$ \\ Departments of ${ }^{1}$ Pain Management and ${ }^{2}$ Pathology, The Affiliated Hospital of \\ Qingdao University, Qingdao, Shandong 266000, P.R. China
}

Received December 7, 2018; Accepted August 1, 2019

DOI: $10.3892 /$ etm.2019.7983

\begin{abstract}
Targeted percutaneous laser disc decompression (T-PLDD) is a minimally invasive technique for the treatment of lumbar disc herniation (LDH). However, the amount of energy required is large and the nerve can be easily damaged. Therefore, this technology requires improvement. The present study aimed to observe the effects of using a modified optical fiber (Mod) in T-PLDD for the treatment of LDH. A retrospective study was conducted using the database of the Affiliated Hospital of Qingdao University (Qingdao, China). In total, 58 patients who received T-PLDD with the Mod between June 2011 and May 2012 were included in the present study. The 10-point numeric rating score, pain rating index and good-to-excellent rating at 3 months $(1.64 \pm 0.97 ; 5.79 \pm 1.57$; $94.8 \%)$ were lower than those at 1 week $(5.12 \pm 1.37 ; 11.52 \pm 1.85$; $74.2 \%)$, and at 1 month $(3.26 \pm 1.41 ; 7.83 \pm 1.31 ; 82.8 \% ; \mathrm{P}<0.05)$ and were maintained for up to 36 months $(1.48 \pm 0.86 ; 4.91 \pm 1.43$; $96.5 \%)$. The Oswestry disability index and 12-item Short Form Health Survey at 6 months $(24.56 \pm 6.78$; 69.40 \pm 5.08$)$ were improved compared with 1 week, 1 month and 3 months, and were maintained for 36 months $(23.10 \pm 6.20 ; 70.89 \pm 5.39)$. The T2 value decreased at 1 week $(76 \pm 8)$ and returned to normal at 3 months $(152 \pm 11)$. Additionally, patients in the Young group ( $<50$ years old) recovered in a shorter period of time than the patients in the Elderly group. In conclusion, the patients stayed in hospital for 3.34 \pm 0.66 days; pain decreased and function increased optimally at 3-6 months and was maintained for 36 months with no serious complications. Individuals $<50$ years old may be more suitable candidates for T-PLDD with the Mod. The Mod should be applied and
\end{abstract}

Correspondence to: Professor Chuansheng Liu, Department of Pain Management, The Affiliated Hospital of Qingdao University, 1677 Wutaishan Road, Huangdao, Qingdao, Shandong 266000, P.R. China

E-mail: chuansheng@126.com

Key words: targeted percutaneous laser disc decompression, lumbar disc herniation, modified optical fiber promoted in T-PLDD, and its use should be considered in the clinical setting.

\section{Introduction}

Lumbar disc herniation (LDH) is a common clinical degenerative discogenic disease, with an incidence rate of 10-20\% (1). The condition is age-associated, which is closely associated with life and work habits, including bad posture (1). Of the patients with LDH, 70-85\% suffer from lower back pain and unilateral leg neurological symptoms $(2,3)$. Clinically, minimally invasive techniques and open surgery are performed for patients with LDH following failure of conservative treatments. Patients diagnosed with LDH with no severe neurologic symptoms, including cauda equina syndrome, pareses or other syndromes that require acute surgical intervention, are suitable candidates for minimally invasive techniques (4). Targeted percutaneous laser disc decompression (T-PLDD) is one such minimally invasive technique that has been used to treat patients with LDH and is cheaper than surgery $(4,5)$. In T-PLDD, energy produced by a laser fired through an optical fiber vaporizes nucleus pulposus tissue to decrease intradisc pressure, thus causing the extruded nucleus pulposus to retract $(4,5)$.

By 2002, 35,000 PLDDs were performed worldwide and were gradually replaced subsequently due to its high complication rates $(4,6)$. For the conventional optical fiber (Con), the refractive index of the outer core is smaller than that of the fiber core, so the energy released radially is similar to that released from a flashlight. This type of radial energy can lead to the vaporization of the nucleus pulposus in one direction, but the surrounding nucleus pulposus is less affected. Therefore, to sufficiently vaporize the nucleus pulposus and to reduce the intradisc pressure, the laser energy required is too high, which may seriously destroy the structure of the nucleus pulposus and accelerate the degeneration of the intervertebral disc (5). A large amount of energy may easily damage the nerve and lead to a number of complications, including aggravated lumbago, endplate inflammation, spinal canal stenosis and even disability $(4,6-8)$.

Therefore, in 2010, the Con was improved so that the energy could gather around the tip of the optical fiber when 
working (unpublished data). This was applied in clinical practice for the treatment of $\mathrm{LDH}$. The present study, to the best of our knowledge, was the first to report the differences between Con and the modified optical fiber (Mod), and verify its use via animal studies. Additionally, a retrospective study was performed to analyze the effects of using Mod in T-PLDD in patients with lumbar disc herniation.

\section{Materials and methods}

Con. The Con used in the present study was a transparent glass fiber that consisted of a fiber core, silicone resin and nylon bushing (from inside to outside; Fig. 1A and C).

Mod. The composition of the Mod was consistent with that of the Con. However, wells in the Mod optical fiber were created irregularly at $2.5 \mathrm{~mm}$ from the tip of the optical fiber (Fig. 1B and D).

Animal study. A total of 60 spinal motor units supplied by the Affiliated Hospital of Qingdao University, consisting of intervertebral discs, two adjacent vertebral bodies, facet joints and intervertebral ligaments, were obtained from 8 mature male domestic pigs (age, 18 months; weight, 150-170 kg). The pigs were kept at a $12 \mathrm{~h}$ light/dark cycle and received water and food ad libitum at room temperature in a $21 \% \mathrm{O}_{2}+78 \% \mathrm{~N}_{2}$ atmosphere. The 60 units were randomly divided into two groups: The Con and Mod groups. Each group included three subgroups according to the different energies (200, 400 and $600 \mathrm{~J} ; \mathrm{n}=10$ ). All protocols were approved by the Institutional Ethics Committee of the Affiliated Hospital of Qingdao University (Qingdao, China).

Under the guidance of the C-arm X-ray device (Posvet-100HF; Poskom Co., Ltd.), the needle paralleling the two adjacent vertebral bodies was inserted from the posterolateral disc and passed through the fibrous ring to the center of the nucleus pulposus. After confirmation of the needle position, the needle core was removed and the optical fiber was inserted (Con in the Con group and Mod in the Mod group). Subsequently, the parameters of the laser apparatus (1064 nm Nd:YAG pulsed laser therapeutic apparatus; Beijing Dongtai Jiguang Technology Co., Ltd) were set (laser pulse width, $0.4 \mathrm{msec}$; duration of the laser each use, $1 \mathrm{sec}$; interval time, $1 \mathrm{sec}$; laser power, $13.5 \mathrm{~W}$; laser pulse repetition frequency, $15 \mathrm{~Hz}$ ). The total energy was set in different groups. After a total energy of laser ablation $(200,400$ or $600 \mathrm{~J})$ was delivered, the procedure was finished. The length and width of the vaporization cavity were measured using a ruler.

Patients. The database of The Affiliated Hospital of Qingdao University (Qingdao, China) was searched to identify the patients who were hospitalized in the Department of Pain Management between June 2011 and May 2012 and received T-PLDD using the Mod. Patients without complete follow-up who lacked any information were excluded from the present study. All 58 patients, the American Society of Anesthesiologists Grade (5) I-III, underwent T-PLDD successfully. There were 26 males and 32 females with an average age of 52.1 \pm 12.9 years (age range, 21-75 years).
The patients were diagnosed with LDH. The following exclusion criteria was applied: Serious heart, lung or kidney diseases, coagulation abnormalities, communication disorders, local or systemic infections, allergies to anesthetics or steroids, previous surgery for LDH, percutaneous endoscopic lumbar discectomy, open lumbar surgery, spinal fracture or tumor, serious disc calcification or pregnancy. The patients provided written informed consent (including consent for publication) to receive T-PLDD. Additionally, patients with free herniated disc fragments were not suitable for PLDD.

The present study was retrospective in nature. The information in the database included the following: General characteristics, modified MacNab grade (9), 10-point numeric rating score (NRS, 0 for no pain and 10 for unbearable pain) for pain (4), oswestry disability index (ODI) (10), pain rating index (PRI) of short-form McGill pain questionnaire (11), 12-item Short Form Health Survey (SF-12) (6), surgery duration, laser energy, disc height and T2 values according to MRI scans (General Electric Company), hospital days, complications and analgesics intake.

Statistical analysis. In the animal study (three experimental repetitions independently), the T2 values of the nucleus pulposus and the length and width of the vaporization cavity were presented as the mean \pm standard deviation. These quantitative data were compared using one-way ANOVA followed by a Least Significant Difference t-test was used.

In the clinical study, the quantitative data, including the NRS, ODI, PRI, SF-12, disc height and T2 values were compared using one-way ANOVA. The count data, including the modified MacNab grade, complications and analgesic intake, were presented as numbers and percentages and were compared using the $\chi^{2}$ test, Fisher's exact test or $\mathrm{R}^{*} \mathrm{C}$ $\chi^{2}$ test. Subsequently, to observe the effect of age on prognosis following surgery, the data were grouped by age and a Student's t-test, repeated data of ANOVA, $\chi^{2}$ test, Fisher's exact test or $\mathrm{R}^{*} \mathrm{C} \chi^{2}$ test were used. $\mathrm{P}<0.05$ was considered to indicate a statistically significant difference. SPSS (v20.0; IBM Corp.) was used to perform statistical analysis.

\section{Results}

Vaporization cavity of nucleus pulposus in the animal study. Postoperatively, the length and width of the vaporization cavity in the Con 400 group $(0.73 \pm 0.09 \mathrm{~cm} ; 0.32 \pm 0.08 \mathrm{~cm})$ were greater than those of the Con 200 group $(0.36 \pm 0.07 \mathrm{~cm}$; $0.17 \pm 0.07 \mathrm{~cm})$. Additionally, the length and width of the vaporization cavity in the Con 600 group $(1.08 \pm 0.14 \mathrm{~cm}$; $0.69 \pm 0.14 \mathrm{~cm})$ were higher than those in the Con 400 group $(\mathrm{P}<0.05$; Fig. 2A-C). The length and width of the vaporization cavity in the Mod 400 group $(0.62 \pm 0.06 \mathrm{~cm} ; 0.51 \pm 0.07 \mathrm{~cm})$ and Mod 600 group $(0.69 \pm 0.10 \mathrm{~cm} ; 0.56 \pm 0.07 \mathrm{~cm})$ were increased compared with those in the Mod 200 group $(0.39 \pm 0.07 \mathrm{~cm}$; $0.30 \pm 0.07 \mathrm{~cm} ; \mathrm{P}<0.05)$; however, there were no significant differences identified between the Mod 400 and Mod 600 groups (Fig. 2D-F). Additionally, the length of the vaporization cavity in the Mod 400 and Mod 600 groups were lower than those in the Con 400 and Con 600 groups $(\mathrm{P}<0.05)$; however, there was no significant difference identified between the Mod 200 and Con 200 groups. The width of the vaporization cavity 

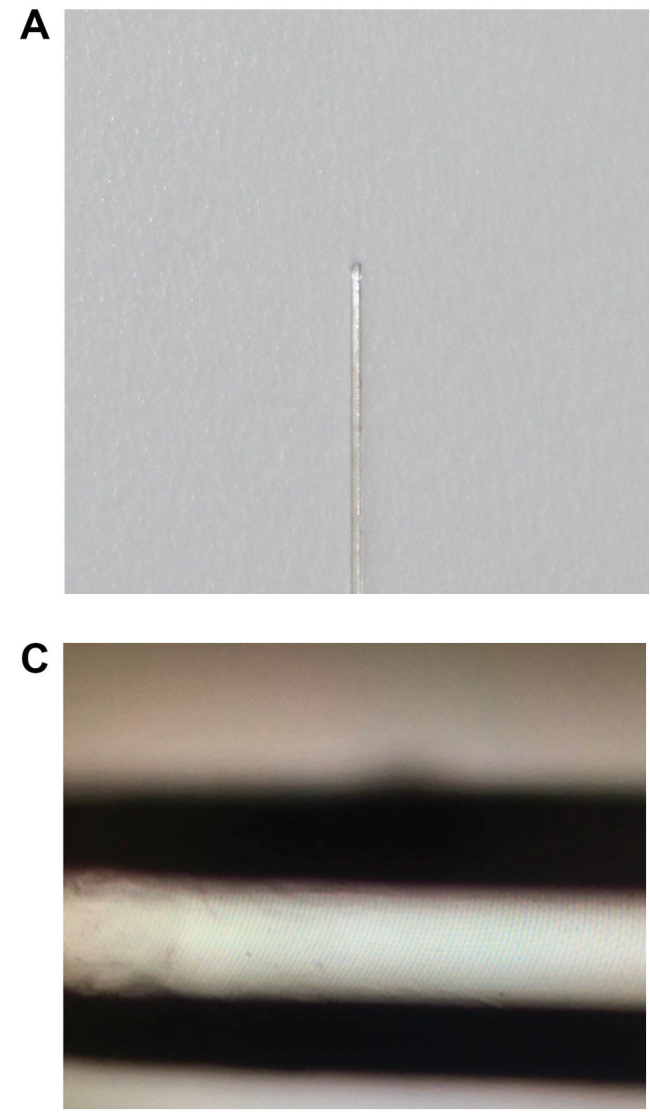

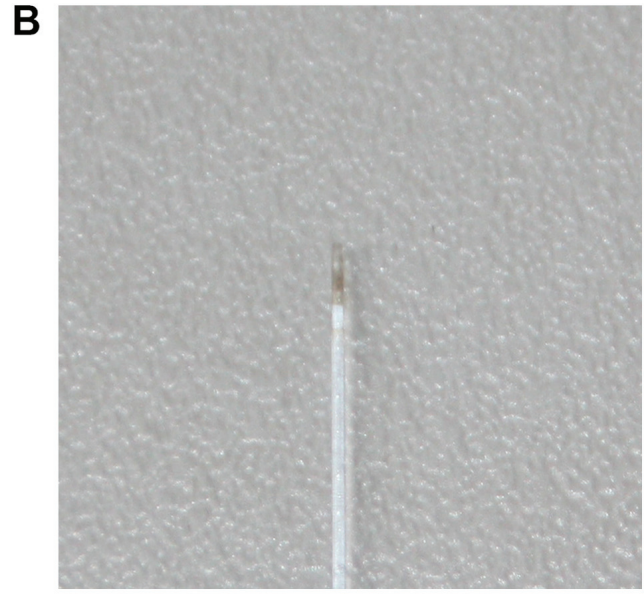

$\mathbf{D}$

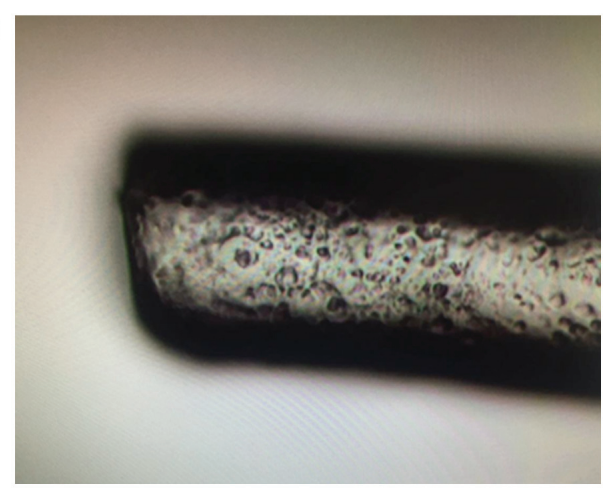

Figure 1. Con and Mod. (A) Macroscopic observations of (A) Con and (B) Mod. (C) Microscopic observation of (C) Con and (D) Mod (magnification, x200). The tip of the Con was smooth, whereas the tip of the Mod was rough and punched irregularly. Con, conventional optical fiber; Mod, modified optical fiber.

in the Mod 200 and Mod 400 groups was higher than that in the Con 200 and Con 400 groups $(\mathrm{P}<0.05)$, whereas that in the Mod 600 group was lower than that in the Con 600 group (P<0.05; Fig. 2G).

Patient characteristics. All patients received drugs and/or physiotherapy, including but not limited to polarized light and ultrasonic drug penetration therapy or epidural block therapy without any previous spinal surgery at the same disc level. Among the patients, 28 and 30 had L4/5 and L5/S1 levels of disc herniation, respectively, and 31 patients had left leg pain, whereas 27 patients had right leg pain. The American Society of Anesthesiologists Grade (5) grades of I, II and III were attributed to 17,31 and 10 patients, respectively. The average duration of pain was $12.1 \pm 5.0$ months (range, 2.7-21.8). Preoperatively, the NRS, ODI, SF-12 and PRI of the patients were $7.86 \pm 1.15$

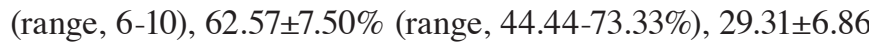
(range, 20.45-43.18) and 15.28 \pm 2.30 (range, 11-19), respectively (Table I).

Surgery-associated results and hospital stay. Under the guidance of radiation, the needle reached one-fifth of the distance into the intervertebral space at the lateral position (Fig. 3A) and at the lateral border of the vertebral body in the anteroposterior position (Fig. 3B). The surgery time was $58.9 \pm 6.6 \mathrm{~min}$ and the average length of hospital stay was $3.34 \pm 0.66$ days. There were 12 patients who received $150 \mathrm{~J}, 19$ patients who received $200 \mathrm{~J}$, nine patients who received $250 \mathrm{~J}$ and 18 patients who received 300 J. No serious complications, including nerve injury and infection, occurred in any of the patients; however, there was one case of hematoma, which resolved within 7 days. There were 19 patients with pain who received Celebrex (200 mg/day) for 2 days (Table II).

NRS, ODI, PRI and SF-12 in patients. The NRS in the patients preoperatively was $7.86 \pm 1.15$ and decreased significantly to $5.12 \pm 1.37$ at 1 week postoperatively, $3.26 \pm 1.41$ at 1 month, $1.64 \pm 0.97$ at 3 months, $1.53 \pm 0.88$ at 6 months, $1.50 \pm 0.86$ at 12 months, $1.48 \pm 0.86$ at 24 months and $1.48 \pm 0.86$ at 36 months $(\mathrm{P}<0.05)$. Compared with the NRS at 1 week, the 1-month score was decreased significantly and the 3-month score was lower than the 1 -month score $(\mathrm{P}<0.05)$. However, the NRS of patients at 3,6,12, 24 and 36 months postoperatively exhibited no significant difference. A similar trend was revealed for the ODI, SF-12 and PRI; however, those of patients at $6,12,24$ and 36 months post-surgery exhibited no significant difference (Table III).

Modified MacNab. The clinical good-to-excellent rating at 1 week post-surgery was $74.2 \%$, which increased significantly to $82.8 \%$ at 1 month and to $94.8 \%$ at 3 months $(\mathrm{P}<0.05)$. The good-to-excellent rating at 6 months reached $96.5 \%$ and was maintained until 36 months. However, no significant differences were identified among the good-to-excellent ratings at 3, 6, 12, 24 and 36 months (Table IV).

Alterations of intervertebral disc height and T2 values after surgery. The intervertebral disc height postoperatively was not 
A

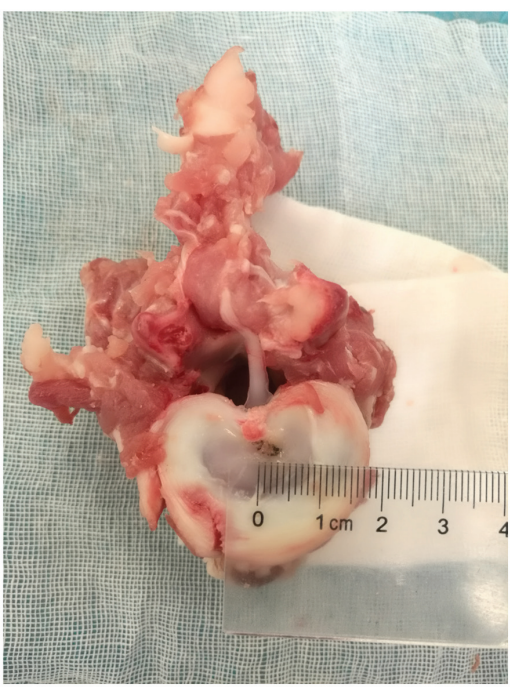

D

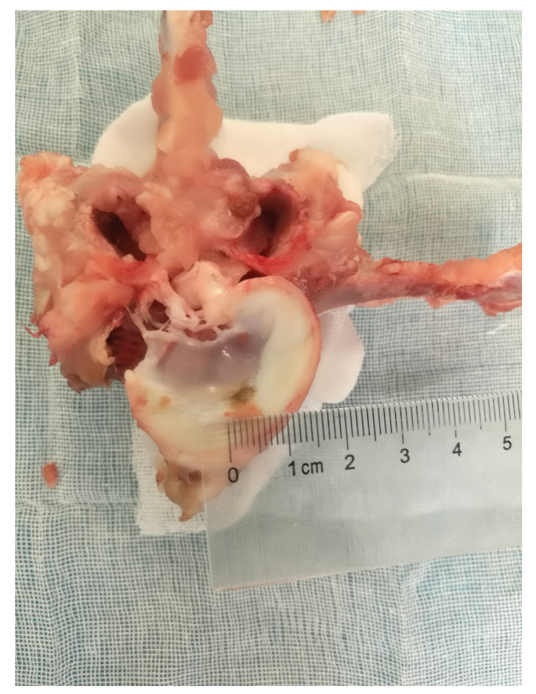

B

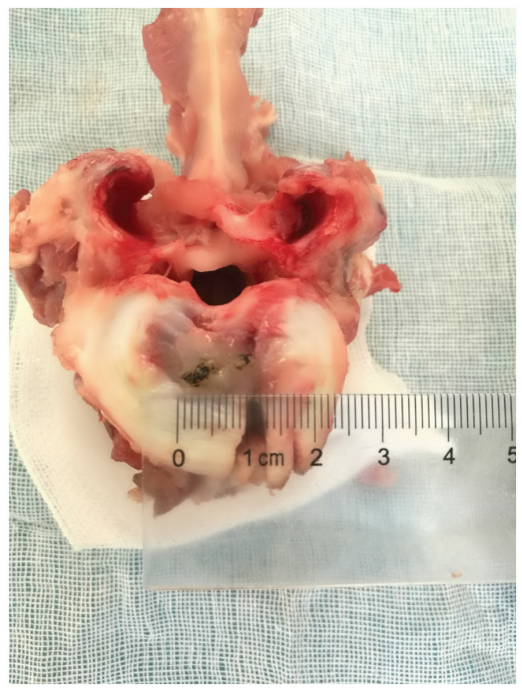

E

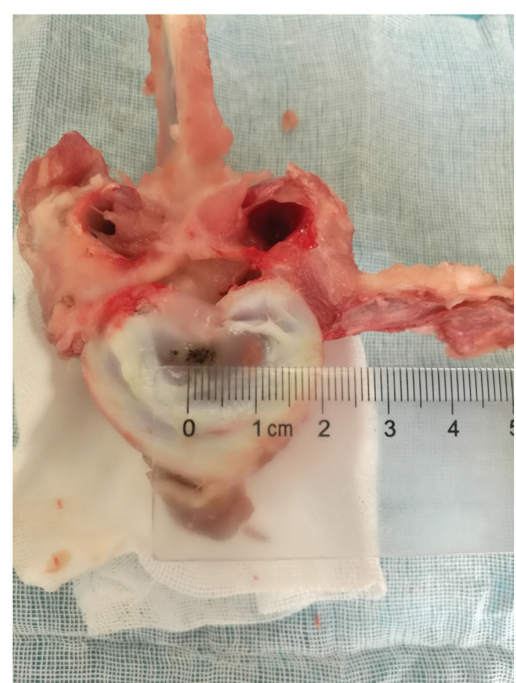

C

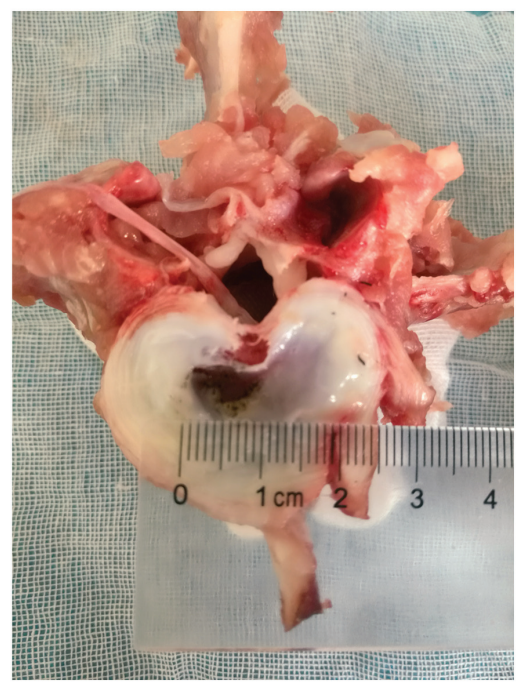

F

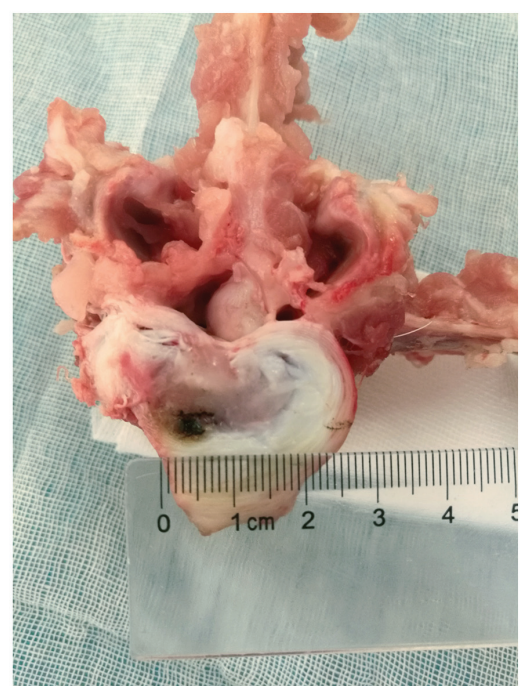

\section{G (cm)}

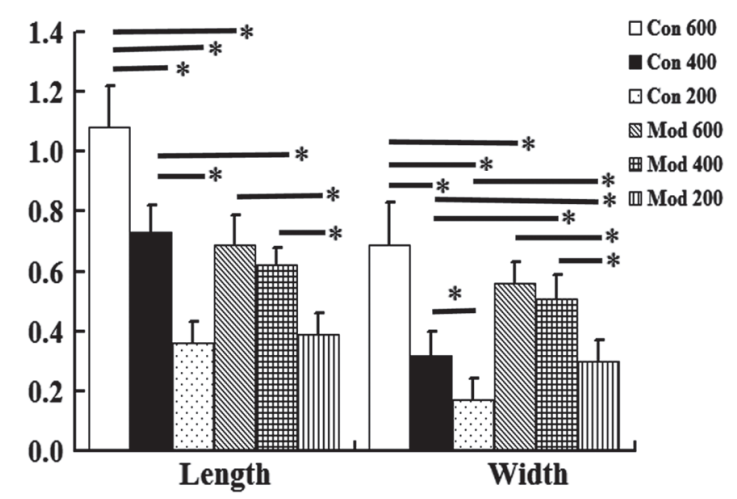

Figure 2. Length and width of the vaporization cavity in pig spinal motor units. (A) Vaporization cavity with (A) $200 \mathrm{~J}$ of a Con, (B) $400 \mathrm{~J}$ of a Con, (C) $600 \mathrm{~J}$ of a Con, (D) $200 \mathrm{~J}$ of a Mod, (E) $400 \mathrm{~J}$ of a Mod and (F) $600 \mathrm{~J}$ of a Mod. (G) Length and width of the vaporization cavity in different groups (n=10). * $<0.05$ as indicated. Con, conventional optical fiber; Mod, modified optical fiber.

identified to exhibit a significant difference compared with the preoperative value (Table V). The T2 value at 1-week postoperatively was $76 \pm 8$, which was lower than the preoperative value $(156 \pm 11 ; \mathrm{P}<0.05)$. However, the $\mathrm{T} 2$ value increased to $152 \pm 11$ at 3 months and was maintained until 36 months, when it exhibited no significant difference compared with the preoperative value (Table V).
Age-associated results. To observe the effect of age on prognosis following surgery, the patients were divided into two groups: The elderly group ( $\geq 50$ years of age) and the young group $(<50$ years of age). There were 33 patients in the Elderly group (18 males and 15 females) and 25 patients in the Young group (eight males and 17 females). The characteristics of the two groups exhibited no significant differences (Table VI). 


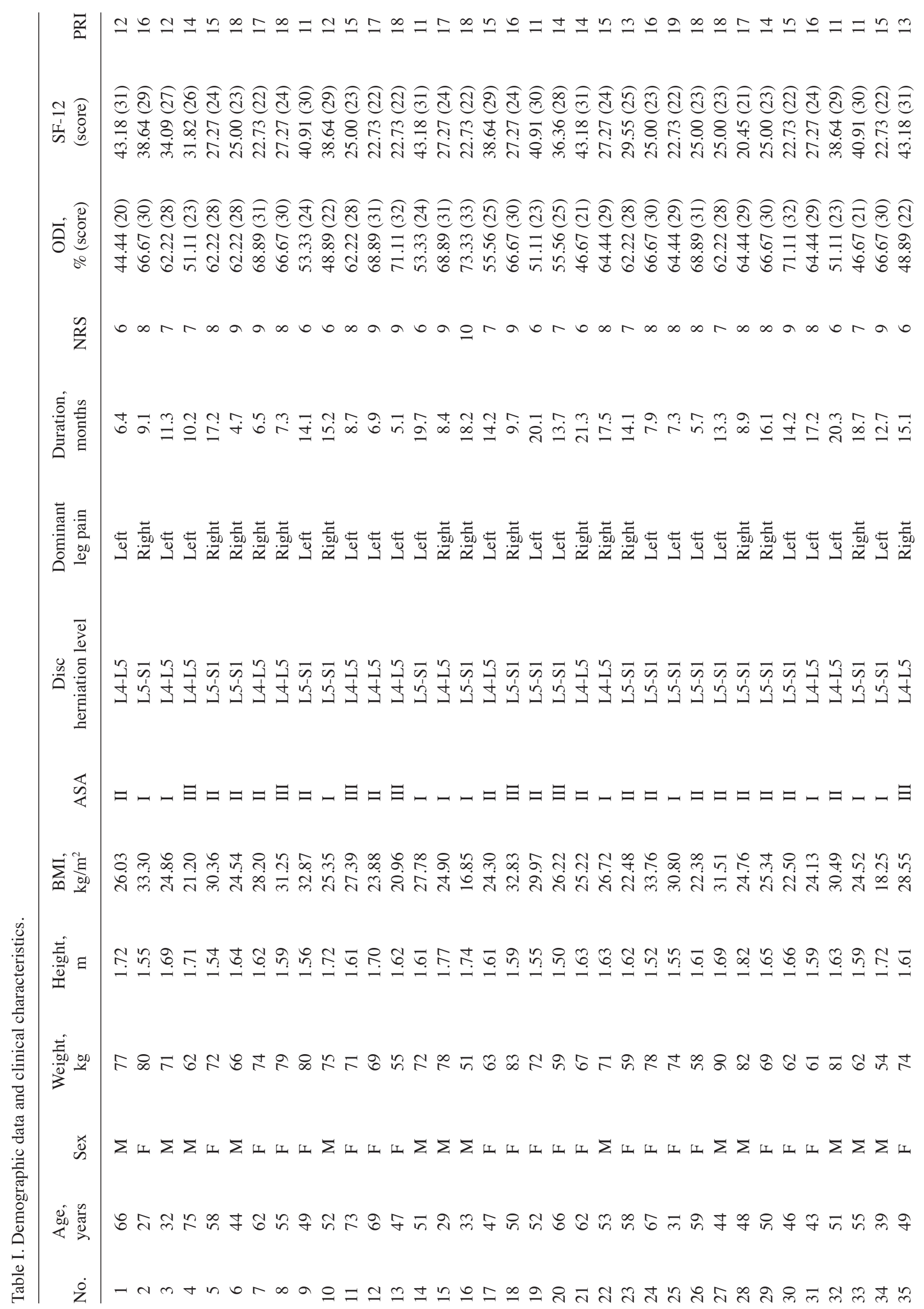




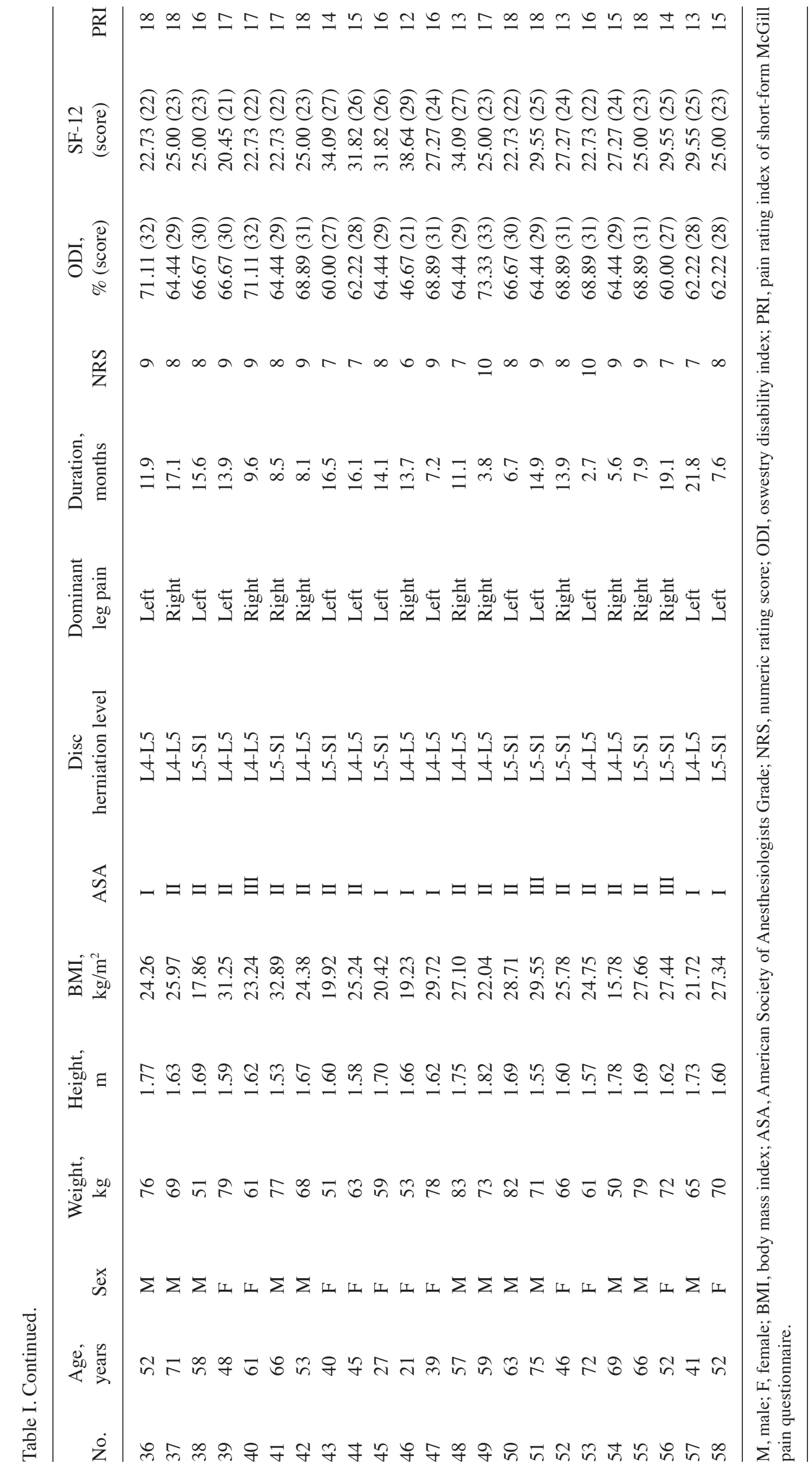


A

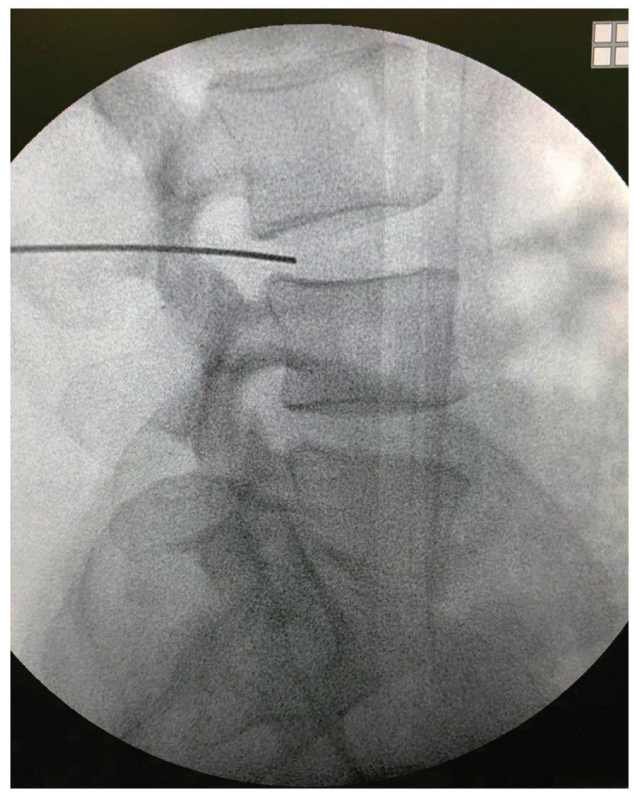

B

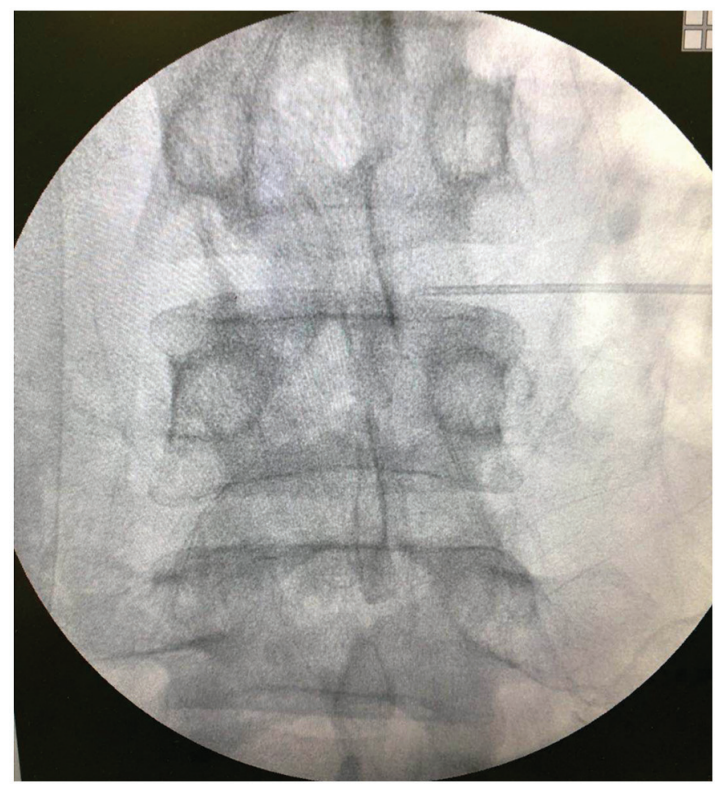

Figure 3. Position of the needle tip in targeted percutaneous laser disc decompression under X-ray guidance. (A) Needle tip located at one-fifth of the distance into the intervertebral space in the lateral position. (B) Needle tip located at the lateral border of the vertebral body in the anteroposterior position.

There was no significant difference in the NRS at different time points between the Young and Elderly groups. In the Elderly group, the NRS at 6 months was lower than that at baseline, 1 week, 1 month and 3 months $(\mathrm{P}<0.05)$, whereas it was similar to that at 12, 24 and 36 months. In the Young group, the NRS at 3 months was lower than that at baseline, 1 week and 1 month $(\mathrm{P}<0.05)$, while it was similar to that at 6,12, 24 and 36 months. The ODI at 12 months in the Elderly group was lower than that at baseline, 1 week, and 1,3 and 6 months $(\mathrm{P}<0.05)$, while it was similar to that at 24 and 36 months. The ODI at 6 months in the Young group was lower than that at baseline, 1 week, 1 month and 3 months $(\mathrm{P}<0.05)$, while it was similar to that at 12,24 and 36 months. The SF-12 results exhibited a similar trend compared with ODI and the PRI results exhibited a similar trend compared with the NRS (Table VII).

\section{Discussion}

At present, T-PLDD has been replaced by other minimally invasive techniques due to the increased rate of complications (12). However, the Con was modified by create wells irregularly at the tip to decrease the complication rate. It was used in the clinical setting for the treatment of LDH to determine the curative effects, which were evaluated in a retrospective study.

The Mod was based on the radiofrequency electric knife. Following modification, energy is released so as to be turned into emission around the tip, and the laser energy is concentrated at the local area. When the radiofrequency is operational, the energy gathers in the tip of the radiofrequency electric knife and only acts on the tissues located around the tip, as indicated in the current study. Therefore, the traditional optical fiber was improved to make its energy gather at the top of the fiber. First, an animal study was performed to observe the effects of the Mod on the vaporization cavity. The length of the vaporization cavity created by the Mod was similar to the width, which increased the volume of the vaporization cavity significantly and further demonstrated that the energy was being evenly released around the tip, not just in a forward direction. Additionally, the Mod exhibited a ceiling effect; the vaporization cavity was altered inconspicuously when the energy exceeded $400 \mathrm{~J}$. This may be due to several reasons. First, the energy spread from the tip of the fiber, whereas the range was not altered for a high energy attenuation rate when the energy exceeded $400 \mathrm{~J}$. Second, the nucleus pulposus was optimally vaporized when the energy reached $400 \mathrm{~J}$. In addition, the water content of the target nucleus pulposus was finite, so no more water was vaporized as the energy increased. Therefore, the $400 \mathrm{~J}$ energy of the Mod provided the greatest effectiveness and the energy did not exceed $400 \mathrm{~J}$ in one position when the Mod was used in a clinical application for T-PLDD.

During surgery, the Mod achieved a more accurate targeted localization without destroying additional tissue. Although $400 \mathrm{~J}$ of the Mod provided the greatest effectiveness in animal studies, $150,200,250$ or $300 \mathrm{~J}$ was also used in the clinical setting. The animal study could not be entirely replicated in the clinical setting, as energies had to be selected based on the tolerance of patients and the degree of extrusion of the nucleus pulposus.

In the clinical study, the Mod used in T-PLDD for patients increased the good-to-excellent rating and decreased the NRS, ODI, PRI and SF-12 in the short- and long-term with no marked alterations observed in intervertebral disc height and $\mathrm{T} 2$ values. The total length of the hospital stay was only $3.34 \pm 0.66$ days.

The present study used $\mathrm{T} 2$ relaxation on a quantitative T2-mapping MRI technique (T2 value) to assess the water content of the nucleus pulposus. The water content of the nucleus pulposus was abundant and the $\mathrm{T} 2$ relaxation on quantitative T2-mapping MR technique of the MRI tended 
Table II. Surgery-associated results and hospital stay.

\begin{tabular}{|c|c|c|c|c|c|c|c|c|c|}
\hline \multirow{2}{*}{$\begin{array}{l}\text { Surgery } \\
\text { time (min) } \\
58.9 \pm 6.6\end{array}$} & \multicolumn{4}{|c|}{ Laser energy } & $\begin{array}{l}\text { Hospital } \\
\text { stay, days }\end{array}$ & \multicolumn{2}{|c|}{ Complications } & \multicolumn{2}{|c|}{ Analgesic } \\
\hline & $150 \mathrm{~J}(\mathrm{n})$ & $200 \mathrm{~J}(\mathrm{n})$ & $250 \mathrm{~J}(\mathrm{n})$ & $300 J(n)$ & $3.34 \pm 0.66$ & Yes (n) & No (n) & Yes (n) & No (n) \\
\hline & 12 & 19 & 9 & 18 & & 1 & 57 & 19 & 39 \\
\hline
\end{tabular}

Table III. Clinical outcomes of the patients in terms of NRS, ODI, SF-12 and PRI.

\begin{tabular}{|c|c|c|c|c|c|c|c|c|}
\hline Variable & Baseline & 1 week & 1 month & 3 months & 6 months & 12 months & 24 months & 36 months \\
\hline VINS & $7.86 \pm 1.15$ & $12 \pm$ & & & & & & \\
\hline ODI (\%) & $62.57 \pm 7.50$ & $44.94 \pm 6.97^{\mathrm{a}}$ & $33.03 \pm 7.23^{\mathrm{a}, \mathrm{b}}$ & $27.70 \pm 7.71^{\mathrm{a}-\mathrm{c}}$ & $24.56 \pm 6.78^{\mathrm{a}-\mathrm{d}}$ & 23.45 & 23.10 & $.20^{\mathrm{a}-\mathrm{d}}$ \\
\hline SF-12 & $29.31 \pm$ & $39.34 \pm 5.27^{\mathrm{a}}$ & $54.90 \pm 4.83^{\mathrm{a}, \mathrm{b}}$ & $64.26 \pm 4.92^{\mathrm{a}-\mathrm{c}}$ & $69.40 \pm 5.08^{\mathrm{a}-\mathrm{d}}$ & $70.57 \pm 5.41^{\mathrm{a}-\mathrm{d}}$ & $70.89 \pm 5.39^{a-d}$ & $70.89 \pm 5.39^{\mathrm{a}-\mathrm{d}}$ \\
\hline PRI & $15.28 \pm 2.30$ & $11.52 \pm 1.85^{\mathrm{a}}$ & $7.83 \pm 1.31^{\mathrm{a}, \mathrm{b}}$ & $5.79 \pm 1.57^{\mathrm{a}-\mathrm{c}}$ & $5.10 \pm 1.51^{\mathrm{a}-\mathrm{d}}$ & $4.93 \pm 1.46^{\mathrm{a}-\mathrm{d}}$ & $4.91 \pm 1.43^{\mathrm{a}-\mathrm{d}}$ & $4.91 \pm 1.43^{\mathrm{a}-\mathrm{d}}$ \\
\hline
\end{tabular}

${ }^{\mathrm{a}} \mathrm{P}<0.05$ vs. Baseline; ${ }^{\mathrm{b}} \mathrm{P}<0.05$ vs. 1 week; ${ }^{\mathrm{c}} \mathrm{P}<0.05$ vs. 1 month; ${ }^{\mathrm{d}} \mathrm{P}<0.05$ vs. 3 months. NRS, numeric rating score; ODI, oswestry disability index; PRI, pain rating index of short-form McGill pain questionnaire; SF-12, the 12-items Short Form Health Survey.

Table IV. Clinical outcomes in terms of modified MacNab.

\begin{tabular}{|c|c|c|c|c|c|c|c|}
\hline MacNab & $\begin{array}{c}1 \text { week, } \\
\text { n }(\%)\end{array}$ & $\begin{array}{l}1 \text { month, } \\
\mathrm{n}(\%)\end{array}$ & $\begin{array}{c}3 \text { months, } \\
\text { n }(\%)\end{array}$ & $\begin{array}{c}6 \text { months, } \\
\mathrm{n}(\%)\end{array}$ & $\begin{array}{c}12 \text { months, } \\
\mathrm{n}(\%)\end{array}$ & $\begin{array}{c}24 \text { months, } \\
\text { n }(\%)\end{array}$ & $\begin{array}{c}36 \text { months, } \\
\mathrm{n}(\%)\end{array}$ \\
\hline Excellent & $27(46.6)$ & $35(60.4)$ & $48(82.8)$ & $50(86.2)$ & $51(87.9)$ & $51(87.9)$ & $51(87.9)$ \\
\hline Good & $16(27.6)$ & $13(22.4)$ & $7(12.0)$ & $6(10.3)$ & $5(8.6)$ & $5(8.6)$ & $5(8.6)$ \\
\hline Fair & $10(17.2)$ & 7 (12.0) & $3(5.2)$ & $2(3.5)$ & $2(3.5)$ & $2(3.5)$ & $2(3.5)$ \\
\hline Poor & $5(8.6)$ & $3(5.2)$ & $0(0)$ & $0(0)$ & $0(0)$ & $0(0)$ & $0(0)$ \\
\hline $\begin{array}{l}\text { Good to } \\
\text { excellent rate }\end{array}$ & $74.2 \%$ & $82.8 \%{ }^{\mathrm{a}}$ & $94.8 \%$ & $96.5 \%$ & $96.5 \%$ & $96.5 \%$ & $96.5 \%{ }^{\mathrm{a}, \mathrm{b}}$ \\
\hline
\end{tabular}

${ }^{\mathrm{a}} \mathrm{P}<0.05$ vs. 1 week; ${ }^{\text {b }} \mathrm{P}<0.05$ vs. 1 month.

Table V. Disc height and T2 value according to MRI.

\begin{tabular}{|c|c|c|c|c|c|c|c|c|}
\hline Variable & Baseline & 1 week & 1 month & 3 months & 6 months & 12 months & 24 months & 36 months \\
\hline Disc height, mm & $12.3 \pm 1.0$ & $12.2 \pm 1.0$ & $12.2 \pm 0.9$ & $12.4 \pm 1.1$ & $12.2 \pm 0.9$ & $12.2 \pm 1.0$ & $12.1 \pm 1.0$ & $12.2 \pm 1.1$ \\
\hline T2 value & $156 \pm 11$ & $76 \pm 8^{a}$ & $110 \pm 10^{\mathrm{a}, \mathrm{b}}$ & $152 \pm 11^{\mathrm{b}, \mathrm{c}}$ & $156 \pm 11^{\mathrm{b}, \mathrm{c}}$ & $155 \pm 11^{\mathrm{b}, \mathrm{c}}$ & $156 \pm 11^{\mathrm{b}, \mathrm{c}}$ & $156 \pm 17^{\mathrm{b}, \mathrm{c}}$ \\
\hline
\end{tabular}

${ }^{\mathrm{a}} \mathrm{P}<0.05$ vs. Baseline; ${ }^{\mathrm{b}} \mathrm{P}<0.05$ vs. 1 week; ${ }^{\mathrm{c}} \mathrm{P}<0.05$ vs. 1 month.

to be a high signal that was reduced when the nucleus pulposus degenerated. Zhu et al (13) revealed that the T2 value exhibits a high sensitivity for detecting water alterations in the nucleus pulposus. Additionally, several studies have demonstrated a good association between the T2 value and water content in the disc $(14,15)$. Therefore, the relevance of the T2 value and water content in the present study were not further validated. As the results demonstrated, the T2 value increased to normal at 3 months post-surgery, which demonstrated that the water content of the nucleus pulposus could be restored after 3 months in patients treated using T-PLDD.

Compared with open surgery, PLDD provides improved long-term outcomes and is safer and less invasive (16). However, the energy required for PLDD is large, and short-term clinical outcomes are not significant (17). Luo et al (6) reported that T-PLDD improves short-term clinical outcomes. The present study also revealed that T-PLDD improved short-term outcomes, as demonstrated by the increased good-to-excellent rating and SF-12 and the decreased NRS, ODI and PRI. In 
Table VI. Characteristics of patients in the Elderly and Yong groups.

\begin{tabular}{lccc}
\hline Characteristic & $\begin{array}{c}\text { Elderly } \\
\text { group }\end{array}$ & $\begin{array}{c}\text { Young } \\
\text { group }\end{array}$ & P-value \\
\hline Sex, n (male/female) & $18 / 15$ & $8 / 17$ & 0.113 \\
ASA & & & 0.094 \\
$\quad$ I & 6 & 11 & \\
II & 20 & 11 & \\
III & 7 & 3 & \\
BMI, kg/m² & $26.13 \pm 3.89$ & $25.38 \pm 4.86$ & 0.519 \\
Disc herniation level, & $17 / 16$ & $11 / 14$ & 0.606 \\
n (L4-L5/L5-S1) & & & \\
Dominant leg pain, & $16 / 17$ & $15 / 10$ & 0.434 \\
n (left/right) & & & \\
Duration, months & $11.7 \pm 5.5$ & $12.7 \pm 4.2$ & 0.471 \\
NRS & $7.82 \pm 1.19$ & $7.92 \pm 1.12$ & 0.741 \\
ODI, \% & $61.82 \pm 8.15$ & $63.56 \pm 6.57$ & 0.387 \\
SF-12 & $29.48 \pm 7.04$ & $63.56 \pm 6.57$ & 0.834 \\
PRI & $15.21 \pm 2.42$ & $15.36 \pm 2.18$ & 0.811 \\
Operation time, min & $58.15 \pm 6.20$ & $59.84 \pm 7.15$ & 0.340 \\
Hospital stay, days & $3.48 \pm 0.91$ & $3.32 \pm 0.69$ & 0.452 \\
\hline
\end{tabular}

ASA, American Society of Anesthesiologists Grade; BMI, body mass index; NRS, numeric rating score; ODI, oswestry disability index; SF-12, the 12-items Short Form Health Survey; PRI, pain rating index of short-form McGill pain questionnaire.

the present study, pain-associated scores including NRS and PRI, were decreased at 1 week and reached their best state at 3 months post-surgery. The functional scores, including ODI and SF-12 were restored to their best state at 6 months post-surgery.

In the study by Luo et al (6), the total laser energy was 600-1500 J forT-PLDD, whichis similar to the non-T-PLDD(17). The rate of reoperation in T-PLDD was $\sim 38 \%$ (18). Although no serious complications have been observed within 1 year after T-PLDD with 600-1500 J (6), it is difficult to evaluate the long-term prognosis. In the present study, a lower energy of 150-300 J was used and there were differences in outcomes compared with the study by Luo et al (6). Luo et al (6) revealed that the Visual Analogue Scale at 3 days, 1 month and 12 months was $2.94 \pm 1.10,2.24 \pm 0.66$ and $1.31 \pm 0.85$, respectively, and the good-to-excellent rating at 12 months was $82.5 \%$, which was similar to the present study. However, the good-to-excellent rating at 1 month $(57.5 \%)$ was markedly lower than that in the present study $(82.8 \%)$. This suggests that the Mod achieved the same therapeutic effects at low energy and provided improved pain relief and a good-to-excellent rating during short- and long-term follow-up.

Zhao et al (4) adopted 300-500 J in the T-PLDD and the good-to-excellent ratings at 1 and 12 months were 80.0 and $92.0 \%$, respectively, which was also indicated in the present study. However, only one index (the modified MacNab) was used in the study by Zhao et al (4), which did not fully evaluate the feasibility and safety of this treatment

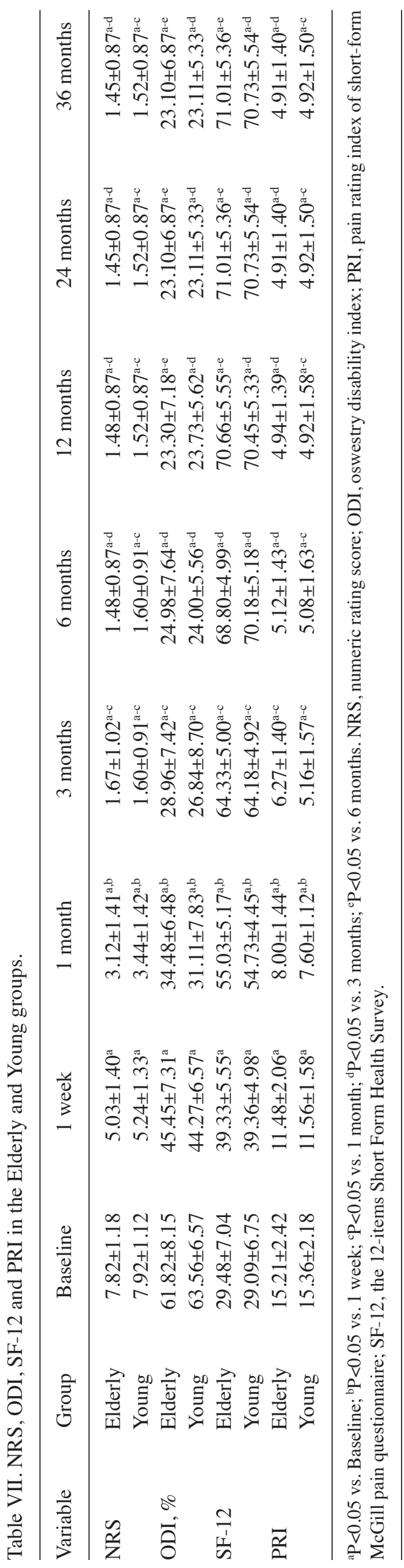


scheme. The present study first demonstrated the ceiling effect of Mod in animal experiments; the energy could not exceed 400 J. Furthermore, the present study reported improved clinical long-term outcomes. Pain relief was maintained for 3 years, as demonstrated by the NRS and PRI, the functional outcomes also indicated an improvement in the 3-year follow-up. No pain recurrence or hypofunction occurred during the 3-year follow-up. Additionally, the intervertebral disc height following surgery was not altered, which maintained the normal tissue structure and assisted the quick postoperative recovery. Therefore, the Mod used for patients with T-PLDD provided a great long-term effect. This provided a theoretical basis and clinical data for the clinical promotion of Mod.

Following surgery, 19 patients received nonsteroidal anti-inflammatory drugs for pain at the puncture site for 2 days, which diminished after the puncture site healed. Hematoma occurred in only 1 patient. Surgery was minimally invasive and the puncture needle was very thin. However, a hematoma could result from the rupture of a vessel during puncture or from a prolonged coagulation time secondary to old age. The hematoma resolved within 7 days and the therapeutic outcome was not affected.

To observe the effects of age on the outcomes of Mod used for T-PLDD, patients were divided into Elderly and Young groups. It was identified that the pain in patients $<50$ years old was most alleviated at 3 months after surgery and perfect recovery of function occurred at 6 months after surgery. For the patients $>50$ years old, the perfect recovery state following surgery was delayed; however, the long-term outcomes were not affected.

The present study had several limitations. First, no record of NRS within 24-48 h after PLDD was found in the database, which was important missing data. However, the patients were prescribed oral antibiotics for 1 day and painkillers were prescribed according to the degree of pain experienced by patients. In the present study, 19 patients received analgesics, which meant that $32.8 \%$ of patients experienced pain within 2 days, which may have been caused by intradiscal, annular inflammation or edema. Second, in the clinical part assessment of the present study, there were no comparison groups, such as a Con group or other treatment groups. Due to this lack of comparison, it was difficult to conclude that the efficacy of Mod was improved compared with that of Con. However, following PLDD with Mod, patients exhibited a satisfactory treatment effect, which meant the PLDD with the Mod was efficacious. Additionally, an advantage of the Mod was that the energy required was lower and the potential complications caused by high energy were avoided. Finally, Ignatieva et al (19) proposed that lasers could induce the damage of degenerative annulus fibrosus of lumbar intervertebral disc. However, their samples were obtained from rabbits and the annulus fibrosus closed to the fiber tip in their study (19). In the present study, only the fiber tip could release energy and the tip was inserted in the nucleus pulposus, which was away from the annulus fibrosus. Therefore, the annulus fibrosus was rarely affected. The observation that the patients were not identified to have significant annulus fibrosus damage during the short- or long-term follow-up also supported this conclusion.
In conclusion, application of the Mod in T-PLDD decreased the pain experienced by patients following LDH and improved the quality of life and curative effects in the short- and long-term. There was no alteration in the intervertebral disc height observed at a relatively low energy during a 3-year follow-up. The Mod should replace the Con in T-PLDD in a clinical setting due to its low energy and good curative effects. Additionally, patients with LDH who are $<50$ years old should be recommended for T-PLDD surgery.

\section{Acknowledgements}

Not applicable.

\section{Funding}

The present study was supported by the Science and Technology Plan of Qingdao (grant no. 3067).

\section{Availability of data and materials}

The datasets used and/or analyzed during the current study are available from the corresponding author on reasonable request.

\section{Authors' contributions}

CM made substantial contributions to conception and design, drafted the manuscript, and approved the version to be published. YL made substantial contributions to acquisition of data, drafted the manuscript and approved the version to be published. SW made substantial contributions to analysis and interpretation of data, completed all animal experiments, drafted the manuscript and approved the final version to be published. JY made substantial contributions to the acquisition of data, drafted the manuscript and approved the version to be published. DK made substantial contributions to analysis and interpretation of data, revised the manuscript critically for important intellectual content and approved the version to be published. CL made substantial contributions to conception and design, provide the grant, drafted the manuscript, revised the manuscript critically for important intellectual content and approved the version to be published.

\section{Ethics approval and consent to participate}

All protocols were approved by the Institutional Ethics Committee of the Affiliated Hospital of Qingdao University (Qingdao, China). The present study was retrospective in nature. The patients provided written informed consent (including consent for publication) to receive T-PLDD.

\section{Patient consent for publication}

The patients provided written informed consent (including consent for publication) to receive T-PLDD.

\section{Competing interests}

The authors declare that they have no competing interests. 


\section{References}

1. Ramakrishnan A, Webb KM and Cowperthwaite MC: One-year outcomes of early-crossover patients in a cohort receiving nonoperative care for lumbar disc herniation. J Neurosurg Spine 7: 391-396, 2017.

2. Ren C, Li Y, Qin R, Sun P and Wang P: Transforaminal endoscopic lumbar discectomy for lumbar disc herniation causing bilateral symptoms. World Neurosurg 106: 413-421, 2017.

3. Andersson GB: Epidemiological features of chronic low-back pain. Lancet 354: 581-585, 1999.

4. Zhao XL, Fu ZJ, Xu YG, Zhao XJ, Song WG and Zheng H: Treatment of lumbar intervertebral disc herniation using C-arm fluoroscopy guided target percutaneous laser disc decompression. Photomed Laser Surg 30: 92-95, 2012.

5. van den Akker-van Marle ME, Brouwer PA, Brand R, Koes B, van den Hout WB, van Buchem MA and Peul WC: Percutaneous laser disc decompression versus microdiscectomy for sciatica: Cost utility analysis alongside a randomized controlled trial. Interv Neuroradiol 23: 538-545, 2017.

6. Luo DX, Jin XJ, Li GT, Sun HT, Li YY and Qi Y: The use of targeted percutaneous laser disc decompression under the guidance of puncture-radiating pain leads to better short-term responses in lumbar disc herniation. Eur Rev Med Pharmacol Sci 18: 3048-3055, 2014.

7. Schenk B, Brouwer PA, Peul WC and van Buchem MA: Percutaneous laser disk decompression: A review of the literature. Am J Neuroradiol 27: 232-235, 2006.

8. Choy DS: Percutaneous laser disc decompression (PLDD): Twelve years' experience with 752 procedures in 518 patients. J Clin Laser Med Surg 16: 325-331, 1998.

9. Oertel JM and Burkhardt BW: Endoscopic intralaminar approach for the treatment of lumbar disc herniation. World Neurosurg 103 : 410-418, 2017.

10. Fairbank JC and Pynsent PB: The oswestry disability index. Spine (Phila Pa 1976) 25: 2940-2952, 2000
11. Melzack R: The short form McGill pain questionnaire. Pain 30: 191-197, 1987.

12. Klessinger S: The frequency of resurgery after percutaneous lumbar surgery using dekompressor in a ten-year period. Minim Invasive Surg 2018: 5286760, 2018.

13. Zhu T, Ai T, Zhang W, Li T and Li X: Segmental quantitative MR imaging analysis of diurnal variation of water content in the lumbar intervertebral discs. Korean J Radiol 16: 139-145, 2015.

14. Perry J, Haughton V, Anderson PA, Wu Y, Fine J and Mistretta C: The value of $\mathrm{T} 2$ relaxation times to characterize lumbar intervertebral disks: Preliminary results. AJNR Am J Neuroradiol 27: 337-342, 2006.

15. Zou J, Yang H, Miyazaki M, Morishita Y, Wei F, McGovern S and Wang JC: Dynamic bulging of intervertebral discs in the degenerative lumbar spine. Spine (Phila Pa 1976) 34: 2545-2550, 2009.

16. Choy DS, Hellinger J, Hellinger S, Tassi GP and Lee SH: 23rd Anniversary of percutaneous laser disc decompression (PLDD). Photomed Laser Surg 27: 535-538, 2009.

17. Choy DS: Percutaneous laser disc decompression: A 17-year experience. Photomed Laser Surg 22: 407-410, 2004.

18. Brouwer PA, Brand R, van den Akker-van Marle ME, Jacobs WC, Schenk B, van den Berg-Huijsmans AA, Koes BW, van Buchem MA, Arts MP and Peul WC: Percutaneous laser disc decompression versus conventional microdiscectomy in sciatica: A randomized controlled trial. Spine J 15: 857-865, 2015.

19. Ignatieva N, Zakharkina O, Andreeva I, Sobol E, Kamensky V and Lunin V: Effects of laser irradiation on collagen organization in chemically induced degenerative annulus fibrosus of lumbar intervertebral disc. Lasers Surg Med 40: 422-432, 2008.

(i) () $९$ This work is licensed under a Creative Commons C. AY NO NO Attribution-NonCommercial-NoDerivatives 4.0 International (CC BY-NC-ND 4.0) License. 